Life Sciences and Medicine

Special Topic: COVID-19: Virus, Immunity and Vaccines

\title{
Challenges of COVID-19 vaccination in patients with cancer
}

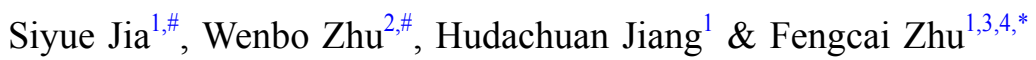 \\ ${ }^{1}$ NHC Key Laboratory of Enteric Pathogenic Microbiology, Jiangsu Provincial Center for Disease Control and Prevention, Nanjing 210009, \\ China; \\ ${ }^{2}$ Jiangsu Cancer Hospital, Affiliated Cancer Hospital of Nanjing Medical University, Nanjing 210009, China; \\ ${ }^{3}$ Center for Global Health (CGH), Nanjing Medical University, Nanjing 211166, China; \\ ${ }^{4}$ Shanghai Institute of Infectious Disease and Biosecurity, Shanghai 200433, China \\ \#Contributed equally to this work. \\ *Corresponding author (email: jszfc@vip.sina.com)
}

Received 9 October 2021; Revised 9 January 2022; Accepted 19 January 2022; Published online 25 March 2022

\begin{abstract}
Patients with cancer are at increased risk of SARA-CoV-2 infection or developing severe COVID-19 cases due to malignancy or immunosuppressive therapy, but they are generally excluded from the target population for COVID-19 vaccination. In general, inactivated vaccines are safe and immunogenic for patients with cancer, but live attenuated vaccines are not recommended. The study suggested that the safety of the mRNA COVID-19 vaccine in patients with cancer is similar to that in healthy people, but immunogenicity is slightly weaker, and a booster dose may be needed. This paper aims to summarize the results of COVID-19 vaccine clinical studies conducted in patients with cancer worldwide and the relevant guidelines released by authorities, so as to provide evidence for promoting COVID-19 vaccination for patients with cancer.
\end{abstract}

Keywords: COVID-19, vaccine, clinical trial, patients with cancer

\section{Introduction}

Cancer is a complex disease which impacts patients including those with active disease, on anticancer treatment, with chronic disease after treatment, or cancer survivors [1]. Of them, immunosuppressed patients are most susceptible to SARS-CoV-2 infection.

A number of studies around the world have reported that patients with COVID-19 with cancer develop a higher risk of severe disease and case fatality rate from SARS-CoV-2 [2,3]. The Chinese Center for Disease Control and Prevention reported a fatality rate of 5.6\% among patients with COVID-19 with cancer [4]. In a retrospective study including 3232 patients in Wuhan, China, the fatality rate in patients with cancer was $29.4 \%$, but that in patients without cancer was only 10.2\% [5]; 17,130 patients with COVID-19 from America diagnosed with hematologic malignancies had worse outcomes (hospitalization: $51.9 \%$, death: $14.8 \%$ ) than those without hematologic malignancies (hospitalization: $23.5 \%$, death: $5.1 \%$ ) [6]; and in Europe, with a fatality rate of $22.5 \%$ in patients with cancer and $14 \%$ in patients without cancer [7].

Patients with cancer who receive systemic anticancer treatments are universally acknowledged as being

(C) The Author(s) 2022. Published by China Science Publishing \& Media Ltd. and EDP Sciences. This is an Open Access article distributed under the terms of the Creative Commons Attribution License (https://creativecommons.org/licenses/by/4.0), which permits unrestricted use, distribution, and reproduction in any medium, provided the original work is properly cited. 
exposed to a higher risk of the disease than those who are not receiving anticancer treatment in consideration of immunosuppressive therapy; however, the evidence to identify it is limited. A prospective observational study in the UK including 800 patients with symptomatic COVID-19 with cancer observed no significant difference in the mortality among patients undergoing chemotherapy and patients undergoing other anticancer treatment [8], and Chinese patients in the early period of epidemic who underwent only radiotherapy did not demonstrate a higher incidence of severe events compared with patients without cancer [9].

Other negative impacts include delaying, suspending or never starting anticancer treatment, pausing or limiting the periodic checking and screening, lower effectiveness of COVID-19 vaccine, and challenged highquality care in the outbreak environment $[10,11]$. In this paper, we aimed to summarize the results of COVID19 vaccine clinical studies conducted on patients with cancer worldwide and the relevant guidelines released by authorities, hoping to provide evidence for promoting COVID-19 vaccination for patients with cancer.

\section{Safety and immunogenicity results of the COVID-19 vaccine in patients with cancer}

Many authorities offer guidelines on vaccination for patients with cancer. However, the data on safety and immunogenicity in patients with cancer are lacking due to their exclusion from original clinical trials. There were few reports on the safety and immunogenicity of vaccines in patients with cancer until the last six months (mid-2021). Only three original articles were found to report safety or immunogenicity results.

Leticia Monin et al. [12] enrolled 151 patients with cancer ( 95 with solid cancer and 56 with haematological cancer) and 54 healthy controls in their study. The immunogenicity analysis showed that the proportion of positive anti-S IgG titres at approximately 21 days following a single dose of vaccination across the three cohorts was $38 \%(26 / 51)$ in patients with solid cancer, $18 \%(10 / 32)$ in patients with haematological cancer, and 94\% (32/34) in healthy controls. Correspondingly, 95\% (18/19), 60\% (3/5), and 100\% (12/12) were seropositive after receiving a booster dose. The safety results showed the vaccine was well tolerated; no toxicities were reported in 54\% (75/140) of patients with cancer versus $38 \%(15 / 40)$ in healthy controls following the first dose of BNT162b2, and 71\% (22/31) versus 31\% (5/16) following the second dose. The authors found that one dose of BNT162b2 vaccine yields poor immunogenicity. Immunogenicity increased significantly in patients with solid cancer within two weeks of a booster dose on day 21 after the first dose.

Hagai Ligumsky et al. [13] conducted a retrospective study which enrolled 326 patients with actively solid tumours and 164 healthy controls following two doses of the BNT162b2 vaccine in Israel. The immunogenicity analysis showed $39(11.9 \%)$ of the patients with cancer were seronegative, compared to 5 $(3.0 \%)$ of the control group $(p=0.001)$. Seronegative proportions were higher in the chemotherapy-treated group $(19,18.8 \%)$ compared to immune checkpoint inhibitor patients $(5,9.1 \%)$ and to those treated with targeted therapy $(1,2.6 \%)(p=0.02)$. Safety results were collected using a questionnaire which showed no severe side effects, neither life threatening nor requiring hospitalization was reported.

Alfred Chung Pui So et al. [14] collected data of vaccine-related adverse events (VRAEs) from 373 patients with cancer. The most common VRAEs within seven days post-vaccination were sore arm (61.7\%), fatigue (18.2\%), and headache (12.1\%). The most common grade 3 VRAE was fatigue (1.1\%). They reported that COVID-19 vaccines in oncology patients have mild reactogenicity, but no immunogenicity analysis was available. 


\section{Guidelines for COVID-19 vaccination in patients with cancer}

For patients with cancer, they are more susceptible to vaccine-preventable diseases due to malignancy or immunosuppressive therapy, such as influenza and pneumonia [15]. Moreover, their impaired immunity will result in a suboptimal immune response or antibody persistence to vaccination compared with the healthy people. Receiving the right vaccine at the appropriate time is crucial to improve safety and immunogenicity of the vaccine in patients with cancer.

In general, inactivated vaccines are safe and immunogenic in patients with cancer, but live attenuated vaccines are not recommended for them, especially those with compromised immune function. As per the recommended immunization schedule released by the Centers for Disease Control and Prevention of the United States in 2021, most of the vaccines that healthy people need are also recommended for immunocompromised people, while live attenuated influenza vaccine, measles, mumps, and rubella vaccine, and varicella vaccine are contraindicated for population with immunocompromised status; children and adolescents with severe combined immunodeficiency are not recommended for receiving rotavirus vaccine as well $[16,17]$.

However, the profile of patients with cancer is complex. There are several types of cancers, which require different therapeutic regimens. Furthermore, the current stage of each patient with cancer is different, some are being treated, some are in progress, some are three- or five-year survivors, whose immune status varies greatly. Therefore, on the basis of following the general principles, it is scientific and feasible to comprehensively determine whether some patients with cancer can be vaccinated and how to be vaccinated according to their own health conditions and the treatments they need to receive, which is considered as the precision immunization strategy [18].

More than 25,000 oncology professionals in the European Society for Medical Oncology (ESMO) have called for COVID-19 vaccination in patients with cancer in response to World Health Organization (WHO) principles and objectives to reduce deaths and disease burden. Patients with active disease or those receiving anticancer treatment deserve additional priority unless they have specific contraindications [11], and they should not be prevented from COVID-19 vaccination in clinical trials of anticancer treatments [19]. Also, healthcare staff caring for patients with cancer are suggested to be prioritized for COVID-19 vaccination in order to provide durable high-quality service and reduce the nosocomial transmission as decreased incidence of nosocomial influenza infections in immunocompromised patients with cancer was associated with increased vaccination rates in healthcare workers [20].

The timing of vaccination depends on the individual therapeutic regimen (Table 1). Ideally, immunization is given prior to systemic treatment. However, if the patient has already started systemic treatment, vaccination during treatment is reasonable but with specific timing considerations for various treatments. Generally, a delay of three to six months is recommended for vaccination postchimeric antigen receptor T (CAR T)-cell therapy, bone marrow stem cell transplantation, or antiCD20 antibody therapy [19,21]. For patients with cancer requiring surgical treatment, vaccination is scheduled at two weeks prior to elective surgery or four weeks after emergency surgery. In regard to patients who receive non-cytotoxic monoclonal antibody chemotherapy, non-cytotoxic immunotherapy chemotherapy, tyrosine kinase inhibitors, polyADP-ribose polymerase (PARP) inhibitors, hormone treatments, radiation therapy or immune checkpoint inhibitors (ICI) alone could receive an approved COVID-19 vaccine at any time during their treatment, but for a combination 
Table 1 COVID-19 vaccination timing for patients with cancer in need of therapy

\begin{tabular}{|c|c|}
\hline Therapy & Timing \\
\hline Non-cytotoxic monoclonal antibody inhibitors & No specific timing consideration \\
\hline \multicolumn{2}{|l|}{ Non-cytotoxic immunotherapy chemotherapy } \\
\hline \multicolumn{2}{|l|}{ Chemotherapytyrosine kinase inhibitors } \\
\hline \multicolumn{2}{|l|}{ Poly-ADP-ribose polymerase (PARP) } \\
\hline \multicolumn{2}{|l|}{ Hormone treatments } \\
\hline \multicolumn{2}{|l|}{ Radiation therapy } \\
\hline \multicolumn{2}{|l|}{ Targeted therapy } \\
\hline \multicolumn{2}{|l|}{ Immune checkpoint inhibitors (ICI) alone } \\
\hline A combination of ICI and chemotherapy & One to three weeks before the treatments \\
\hline \multicolumn{2}{|l|}{ Surgical treatment } \\
\hline Elective surgery & Two weeks prior \\
\hline Emergency surgery & Four weeks after \\
\hline Haematopoietic stem cell transplantation (allogenic or autologous) & Three to six months after \\
\hline CAR-T cell therapy or bone marrow & Three to six months after \\
\hline Anti-CD20 antibody therapy & Six months after \\
\hline
\end{tabular}

of chemotherapy and ICI, or cytotoxic chemotherapy, the first dose of vaccine is ideally administered one to three weeks before the special treatments [21,22].

People with certain cancers or who are receiving treatment that suppresses the immune system have a weaker response to COVID-19 vaccines than healthy people with normal immune systems. Centers for Disease Control and Prevention of the United States have suggested that people whose immune systems are moderately to severely compromised and who received the primary immunization - two doses of mRNA COVID-19 vaccine, BNT162b2 or mRNA1273 - are recommended another dose of the same vaccine. According to Centers for Disease Control and Prevention of the United States, such people include those who are being treated for blood cancer (leukaemia, lymphoma, or multiple myeloma), who have received a stem cell transplant within the past two years, and who are taking medicine that may suppress immune response; however, for people with breast, lung, or colon cancers, an additional dose is not allowed to be given. However, there is limited evidence to recommend an additional dose of Ad26.COV2.S [23,24].

\section{Considerations of COVID-19 vaccination for patients with cancer}

In recent decades, some vaccine clinical studies have been conducted to evaluate the safety and effectiveness of vaccines in patients with cancer, especially the seasonal influenza vaccines (trivalent inactivated) and pneumonia vaccines (conjugate and polysaccharide) [25-29]. At present, COVID-19 pandemic caused by SARS-CoV-2 is continuing to scale up all over the world, and large-scale vaccination campaigns against COVID-19 are underway. However, patients with cancer, who are at a high risk of SARS-CoV-2 infection and developing into severe COVID-19 cases, are not the target population of the COVID-19 vaccination campaign. As American Society of Clinical Oncology suggested, people with cancer (or with a history of cancer) can get some vaccines, but this depends on many factors, such as the type of vaccine, the type of cancer a person has (had), if they are still being treated for cancer, and if their immune system is working 
properly.

Up to now, several clinical trials of the mRNA COVID-19 vaccine have been conducted in immunocompromised populations, which showed good safety and acceptable immunogenicity, and an additional dose or mixed-dose series may contribute to superior immunogenicity. Nevertheless, a few of these studies involved patients with cancer and the sample size is small, which need further research. Besides, clinical data on other types of COVID-19 vaccines in immunocompromised population are still lacking, such as the inactivated and protein subunit COVID-19 vaccines.

Both Leticia Monin and Hagai Ligumsky's studies showed that the seropositive rate of the cancer patient group was lower than that of healthy controls. It may be because of an age mismatch between the two groups, and the age of patients with cancer is generally higher ( $70 \%$ of all malignancy-related deaths occur in people 65 years of age or older) [30]. The immune system alterations in the elderly are comprehensively known as immunosenescence [31], which plays an essential role in promoting cancer immunoediting [32], and ignoring this issue may cause the effectiveness of vaccination for patients with cancer to be underestimated compared with the healthy controls.

With worsening symptoms and receiving immunosuppressive therapy, patients with cancer are more susceptible to vaccine-preventable infections. At present, the treatment methods of solid cancer are surgery, chemotherapy, radiotherapy, molecular targeted therapy and immunotherapy, which not only can prolong a patient's life, but also increase the risk of infection, cardiovascular disease, and others [33]. Due to the lack of adequate clinical data and operating guidelines, oncologists often do not actively recommend patients to be vaccinated, the immunization rates remain low in patients with cancer [34]. Solid-organ transplant recipients' data suggest that an additional COVID-19 vaccine dose in immunocompromised people enhances antibody response and increases the proportion who respond [35]. Roni Bitterman et al. [36] assessed the effectiveness of influenza vaccine in immunosuppressed adults with malignancies. The results of their observational data demonstrated a significant association between vaccine receipt and lower risk of death, and no life-threatening or persistent adverse effects of vaccination were reported.

The results of COVID-19 vaccination in patients with cancer conducted in several developed countries provide a good reference for developing countries including China. However, due to the particularity of patients with cancer, there are bound to be many difficulties and obstacles on the way to explore the immunization strategies for them. The following aspects are suggested to promote COVID-19 vaccination in patients with cancer. First, research institutions should actively conduct clinical trials of COVID-19 vaccines among patients with cancer. Based on sufficient clinical trials and data, the government can formulate the policies for promoting vaccination, and the clinical doctors have enough evidence to encourage patients with cancer for vaccination. Second, the government should formulate relevant policies and regulations on vaccination of patients with cancer. The impact of national policies on the COVID-19 vaccination is huge and has strong guidance. Therefore, we hope that the government can take into account the risk of SARS-CoV-2 infection and the heavy disease burden faced by patients with cancer and promote policies and regulations so that the COVID-19 vaccination in patients with cancer can continue with the support of the country and the government. Third, the doctor in charge of patients with cancer should encourage them and their families to receive COVID-19 vaccines. Doctors' advice can be very influential for patients with cancer; based on the results of clinical trials and national policies, medical personnel should communicate with patients and their families to receive the appropriate type of COVID-19 vaccine to reduce the infection rate, thus prolonging 
survival and improving the quality of life. Fourth, the promotion of COVID-19 vaccination in patients with cancer needs the cooperation of multiple departments. Science and technology departments should vigorously support the establishment of projects and encourage the application of relevant research. Health authorities should update vaccination guidelines in a timely manner according to domestic and international research progress. In the medical and health sector, the industry can try off-label use; if the effect of off-label use is verified and consensus is reached, it will accelerate the standardization process of COVID-19 vaccination for special populations.

\section{Data availability}

All data in this article are from published studies.

\section{Conflict of interest}

No potential conflicts of interest were disclosed.

\section{References}

1 ESMO. COVID-19 vaccination in cancer patients: ESMO Statements. https://www.esmo.org/covid-19-and-cancer/ covid-19-vaccination (4 October 2021, date last accessed).

2 He Y, Ding Y, Cao B, et al. COVID-19 vaccine development from the perspective of cancer patients. Hum Vaccines Immunother 2021; 17: 3281-3287.

3 Ribas A, Sengupta R, Locke T, et al. Priority COVID-19 vaccination for patients with cancer while vaccine supply is limited. Cancer Discov 2021; 11: 233-236.

$4 \mathrm{Wu}$ Z, McGoogan JM. Characteristics of and important lessons from the coronavirus disease 2019 (COVID-19) outbreak in China. JAMA 2020; 323: 1239.

5 Meng Y, Lu W, Guo E, et al. Cancer history is an independent risk factor for mortality in hospitalized COVID-19 patients: a propensity score-matched analysis. J Hematol Oncol 2020; 13: 75.

6 Wang QQ, Berger NA, Xu R. When hematologic malignancies meet COVID-19 in the United States: infections, death and disparities. Blood Rev 2021; 47: 100775.

7 Rüthrich MM, Giessen-Jung C, Borgmann S, et al. COVID-19 in cancer patients: clinical characteristics and outcomean analysis of the LEOSS registry. Ann Hematol 2021; 100: 383-393.

8 Lee LY, Cazier JB, Angelis V, et al. COVID-19 mortality in patients with cancer on chemotherapy or other anticancer treatments: a prospective cohort study. Lancet 2020; 395: 1919-1926.

9 Dai M, Liu D, Liu M, et al. Patients with cancer appear more vulnerable to SARS-COV-2: a multi-center study during the COVID-19 outbreak. Cancer Discov 2020; 10: 783-791.

10 Bakouny Z, Paciotti M, Schmidt AL, et al. Cancer screening tests and cancer diagnoses during the COVID-19 pandemic. JAMA Oncol 2021; 7: 458.

11 Garassino MC, Vyas M, de Vries EGE, et al. The ESMO call to action on COVID-19 vaccinations and patients with cancer: Vaccinate. Monitor. Educate. Ann Oncol 2021; 32: 579-581.

12 Monin L, Laing AG, Muñoz-Ruiz M, et al. Safety and immunogenicity of one versus two doses of the COVID-19 vaccine BNT162b2 for patients with cancer: interim analysis of a prospective observational study. Lancet Oncol 2021; 22: 765-778.

13 Ligumsky H, Safadi E, Etan T, et al. Immunogenicity and safety of the BNT162b2 mRNA COVID-19 vaccine among actively treated cancer patients. J Natl Cancer Inst 2021; 114: 203-209.

14 So ACP, McGrath H, Ting J, et al. COVID-19 vaccine safety in cancer patients: a single centre experience. Cancers 2021; 13: 3573. 
15 Charshafian S, Liang SY. Rapid fire: infectious disease emergencies in patients with cancer. Emerg Med Clin $N$ Am 2018; 36: 493-516.

16 Recommended Adult Immunization Schedule for ages 19 years or older, United States. https://www.cdc.gov/vaccines/ schedules/downloads/adult/adult-combined-schedule.pdf (2 October 2021, date last accessed).

17 Recommended Child and Adolescent Immunization Schedule for ages 18 years or younger, United States. https://www. cdc.gov/vaccines/schedules/downloads/child/0-18yrs-child- combined-schedule.pdf (2 October 2021, date last accessed).

18 Jia S, Li J, Liu Y, et al. Precision immunization: a new trend in human vaccination. Hum Vacc Immunother 2020; 16: $513-522$.

19 ESMO. ESMO Statements on vaccination against COVID-19 in people with cancer. https://www.esmo.org/covid-19and-cancer/covid-19-vaccination (6 October 2021, date last accessed).

20 Frenzel E, Chemaly RF, Ariza-Heredia E, et al. Association of increased influenza vaccination in health care workers with a reduction in nosocomial influenza infections in cancer patients. Am J Infect Control 2016; 44: 1016-1021.

21 Gundavda MK, Gundavda KK. Cancer or COVID-19? A review of recommendations for COVID-19 vaccination in cancer patients. Curr Treat Options Oncol 2021; 22: 95.

22 Gauci ML, Coutzac C, Houot R, et al. SARS-CoV-2 vaccines for cancer patients treated with immunotherapies: Recommendations from the French society for ImmunoTherapy of Cancer (FITC). Eur J Cancer 2021; 148: $121-123$.

23 CDC. COVID-19 vaccines for moderately or severely immunocompromised people. https:// www.cdc.gov/coronavirus/ 2019-ncov/vaccines/recommendations/immuno.html (6 October, date last accessed).

24 CDC. Data and clinical considerations for additional doses inimmunocompromised people. https://www.cdc.gov/ vaccines/acip/meetings/downloads/slides-2021-07/07-COVID-Oliver-508.pdf (6 October, date last accessed).

25 Meerveld-Eggink A, de Weerdt O, van der Velden AMT, et al. Response to influenza virus vaccination during chemotherapy in patients with breast cancer. Ann Oncol 2011; 22: 2031-2035.

26 McManus M, Frangoul H, McCullers JA, et al. Safety of high dose trivalent inactivated influenza vaccine in pediatric patients with acute lymphoblastic leukemia. Pediatr Blood Cancer 2014; 61: 815-820.

27 Doganis D, Kafasi A, Dana H, et al. Immune response to influenza vaccination in children with cancer. Hum Vacc Immunother 2018; 14: 2310-2317.

28 Berglund A, Willén L, Grödeberg L, et al. The response to vaccination against influenza A(H1N1) 2009, seasonal influenza and Streptococcus pneumoniae in adult outpatients with ongoing treatment for cancer with and without rituximab. Acta Oncol 2014; 53: 1212-1220.

29 Cheng FWT, Ip M, Chu YYL, et al. Humoral response to conjugate pneumococcal vaccine in paediatric oncology patients. Arch Dis Child 2012; 97: 358-360.

30 Provinciali M. Immunosenescence and cancer vaccines. Cancer Immunol Immunother 2009; 58: $1959-1967$.

31 Yancik R, Ries LAG. Aging and cancer in America. Hematol Oncol Clin North Am 2000; 14: 17-23.

32 Mazzola P, Radhi S, Mirandola L, et al. Aging, cancer, and cancer vaccines. Immun Ageing 2012; 9: 1-11.

33 Baniyash M. Chronic inflammation, immunosuppression and cancer: new insights and outlook. Semin Cancer Biol 2006; 16: 80-88.

34 Ariza-Heredia EJ, Chemaly RF. Practical review of immunizations in adult patients with cancer. Hum Vacc Immunother 2015; 11: 2606-2614.

35 Kamar N, Abravanel F, Marion O, et al. Three doses of an mRNA Covid-19 vaccine in solid-organ transplant recipients. N Engl J Med 2021; 385: 661-662.

36 Bitterman R, Eliakim-Raz N, Vinograd I, et al. Influenza vaccines in immunosuppressed adults with cancer. Cochrane Database Syst Rev 2018; 2: CD008983. 